\title{
ARTICLE
}

\section{Nanoscale electron transport at the surface of a topological insulator}

\author{
Sebastian Bauer ${ }^{1} \&$ Christian A. Bobisch ${ }^{1}$
}

The use of three-dimensional topological insulators for disruptive technologies critically depends on the dissipationless transport of electrons at the surface, because of the suppression of backscattering at defects. However, in real devices, defects are unavoidable and scattering at angles other than $180^{\circ}$ is allowed for such materials. Until now, this has been studied indirectly by bulk measurements and by the analysis of the local density of states in close vicinity to defect sites. Here, we directly measure the nanoscale voltage drop caused by the scattering at step edges, which occurs if a lateral current flows along a three-dimensional topological insulator. The experiments were performed using scanning tunnelling potentiometry for thin $\mathrm{Bi}_{2} \mathrm{Se}_{3}$ films. So far, the observed voltage drops are small because of large contributions of the bulk to the electronic transport. However, for the use of ideal topological insulating thin films in devices, these contributions would play a significant role.

\footnotetext{
${ }^{1}$ Faculty of Physics and Center for Nanointegration Duisburg-Essen (CENIDE), University of Duisburg-Essen, Lotharstrasse 1, 47057 Duisburg, Germany. Correspondence and requests for materials should be addressed to C.A.B. (email: christian.bobisch@uni-due.de).
} 
B oth, the high conductivity and the spin polarization of the topological surface state imply the use of three-dimensional topological insulators (3D TIs) ${ }^{1,2}$ for spintronic devices (for example, for data processing). In addition, the low-energy dissipation due to the forbidden backscattering ${ }^{3-6}$ increases the efficiency and the lifetime of potential TI-based devices. $\mathrm{Bi}_{2} \mathrm{Se}_{3}$ is such a 3D TI with a bulk band gap of about $0.3 \mathrm{eV}$ while the corresponding topological surface state is found to exhibit a nearly ideal Dirac cone ${ }^{7}$. The height of the band gap suppresses intrinsic conduction, so that $\mathrm{Bi}_{2} \mathrm{Se}_{3}$ is well suited for possible technical applications. In addition, the doping of $\mathrm{Bi}_{2} \mathrm{Se}_{3}$ can be tailored by low-level substitutions of $\mathrm{Ca}$ for $\mathrm{Bi}$ to adjust the Fermi level ${ }^{8}$. However, owing to unintentional doping by Se defects, the bulk of thin $\mathrm{Bi}_{2} \mathrm{Se}_{3}$ films is conductive. So far, the global sheet conductance of $\mathrm{Bi}_{2} \mathrm{Se}_{3}$ for different sample preparations has been studied on a macroscopic scale ${ }^{9-12}$. The microscopic scattering in (topological) surface states is mostly analysed by evaluating the lateral variation of the local density of states (LDOSs) using $\mathrm{d} I / \mathrm{d} V$ measurements with a scanning tunnelling microscope $(\mathrm{STM})^{3-5,13}$. Here, electron scattering at defects, for example, surface step edges is measured indirectly by analysing the lateral oscillations of the LDOS near the scattering centre. As this technique provides a high spatial resolution on the atomic scale $e^{14}$, the involved scattering channels are analysed with great spatial resolution $6,15-17$. This has also been supported by theoretical studies $^{15,16,18,19}$, which proved that scattering at step edges on a topological surface is possible without violating the forbidden backscattering, decreasing the electron transport. Very recently, ab-initio calculations reveal the impact of $\mathrm{Bi}_{2} \mathrm{Se}_{3}$ step edges, that is, surface barriers on the electron transport ${ }^{19}$. For TI-based devices, local information on the electron transport through thin TI films on supporting substrates are relevant as processed device structures have reached dimensions of $\sim 14 \mathrm{~nm}$ already. For a 3D TI holding a surface current, the spatial variation of the electrochemical potential $\mu_{\mathrm{ec}}$ at the surface carries direct and detailed information on the electron transport properties and the corresponding energy dissipation ${ }^{20}$. Datta shows that a local scatterer such as a step edge represents a one-dimensional Landauer-like resistive dipole, which results in a local drop of $\mu_{\mathrm{ec}}$, that is, a local voltage drop at the defect site ${ }^{20}$. At the surface of a TI this has not been measured so far.

In the following, we use scanning tunnelling potentiometry (STP $)^{21,22}$ to detect simultaneously $\mu_{\mathrm{ec}}$ and the corresponding

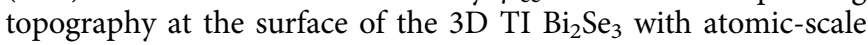
resolution. The analysis of $\mu_{\mathrm{ec}}$ at the thin $\mathrm{Bi}_{2} \mathrm{Se}_{3}$ film surface yields a direct measure of current transport under realistic conditions, that is, a current of a few $\mathrm{mA}$ flows parallel to the surface analogue to real devices. We use thin films of $\mathrm{Bi}_{2} \mathrm{Se}_{3}$ on the technologically important substrate silicon with a film thickness of $14 \mathrm{QL}$, which ensures that the topological state is well established $^{23}$, whereas the surface-to-bulk ratio of the film is still very high. This guarantees a maximum surface sensitivity. In particular, we find that the nanoscale electron transport in $\mathrm{Bi}_{2} \mathrm{Se}_{3}$ thin films is sensitive to surface step edges, manifested in local voltage drops at the step edge positions.

\section{Results}

Sample characterisation by macroscale transport measurements. A sketch of the experimental setup and a SEM image of the contact geometry are shown in Fig. 1a,b. For further experimental details see the Methods. Figure 1c shows a typical STM image of the thin $\mathrm{Bi}_{2} \mathrm{Se}_{3}$ film. It reveals a layered structure with a step height for 1 QL of $1.01 \pm 0.07 \mathrm{~nm}$ (see also Fig. 2d) in agreement with other published data for $\mathrm{Bi}_{2} \mathrm{Se}_{3}$ (ref. 24). The inset in Fig. 1c shows the corresponding sharp and hexagonal low-energy electron diffraction (LEED) pattern indicating a high quality of the epitaxial film with a lateral lattice constant of $0.41 \pm 0.01 \mathrm{~nm}$. Using a multi probe STM, we evaluate the macroscopic conductance of the sample in situ: Two tips contact the $\mathrm{Bi}_{2} \mathrm{Se}_{3}$ surface for different tip distances and the resistance between the tips is measured (inset Fig. 1d). Fitting of the data yields a (macroscopic) sheet conductance $G_{\text {macro }}$ of the film of $1.8 \pm 0.1 \mathrm{mS}$ (Fig. 1d), which agrees to the findings by other groups (for example, $1.3 \mathrm{mS}$ for $10 \mathrm{QL} \mathrm{Bi}_{2} \mathrm{Se}_{3}$ on sapphire (0001), extracted from the diagram in ref. 25). This is discussed in detail in Supplementary Note 1 (see also Supplementary Fig. 1).

Nanoscale transport measurements by STP. Figure 2 shows our experimental STP results, that is, an STM topography (Fig. 2a) and a simultaneously acquired map of the electrochemical potential (for short now called potential; Fig. 2b). Here, a lateral current of $1.9 \mathrm{~mA}$ is flowing through the sample from right to left (direction of the electrons). The potential mainly exhibits a constant gradient along this direction (Fig. 2c and line profile in Fig. 2e), which corresponds to an electric field $E$ of $72 \pm 4 \mathrm{~V} \mathrm{~cm}^{-1}$. This constant gradient may result from phonon scattering as the dominant process within the $\mathrm{Bi}_{2} \mathrm{Se}_{2}$ film, which is not forbidden by the topological phase ${ }^{26,27}$.

For the given geometry, the average local current density $j$ in the middle between the contacting tips can be estimated from the total transverse current $I_{\text {trans }}$ by ${ }^{28}$

$$
j=\frac{2}{\pi \cdot d} I_{\text {trans }},
$$

where $d$ is the distance between the contact tips (for details see Supplementary Note 2 and Supplementary Fig. 2). Given the transverse current of $I_{\text {trans }}=1.9 \mathrm{~mA}$ and the distance between the contacting tips of $d=80 \pm 20 \mu \mathrm{m}$ (see Fig. $1 \mathrm{~b}$ for details), the average local current density is $j=0.15 \pm 0.03 \mathrm{~A} \mathrm{~cm}^{-1}$. Using Ohm's law, we calculate the local (microscopic) sheet conductance $G_{\text {micro }}$ of the film: $G_{\text {micro }}=j / E=2.1 \pm 0.6 \mathrm{mS}$, which is close to the global film conductance of $G_{\text {macro }}=1.8 \pm 0.1 \mathrm{mS}$ as determined from the resistance measurement at macroscopic distances (see Fig. 1d for details). This implies a rather homogeneous sheet conductance of the $\mathrm{Bi}_{2} \mathrm{Se}_{3}$ film. The sheet conductance $G$ of a film is defined as the sum of the film's bulk conductivity $\sigma_{\mathrm{b}}$ times the film thickness $d$ and the surface contribution $\sigma_{\mathrm{s}}$ :

$$
G=\sigma_{\mathrm{b}} \cdot d+\sigma_{\mathrm{s}} .
$$

From literature $9,10,12$, we know that the surface conductivity of high-quality $\mathrm{Bi}_{2} \mathrm{Se}_{3}$ films is in the range of $0.4-0.8 \mathrm{mS}$. Thus, the dominating part $(60-80 \%)$ for the microscopic conductance of our $\mathrm{Bi}_{2} \mathrm{Se}_{3}$ film is the bulk conductivity. This is plausible since during the film growth the Se excess was not very high (around 50\% more Se atoms than necessary) and Se vacancies are incorporated into the film leading to an $\mathrm{n}$-doping ${ }^{8,9}$. In consequence, around $30 \%$ of the current density flows through the surface state.

To analyse the local potential in detail, we subtract the macroscopic gradient from the measured potential and an additional 'fine structure' in the potential becomes visible (Fig. 2c and line profile in Fig. 2d). This 'fine structure' exhibits small voltage drops in the order of $20-30 \mu \mathrm{V}$, which are directly correlated to step edges in the topography (see Fig. 2a,b for details). To emphasise this, Fig. $2 d$ shows line profiles across the same region for the topographic image, the potential image and the 'fine structure'. In addition, we show the line profile for the 'fine structure' for both, forward and backward scan direction. As the potential for both scan directions agree well, we can rule out a measurement artefact due to a non-ideal operating feedback loop for the tunnelling current. 
a

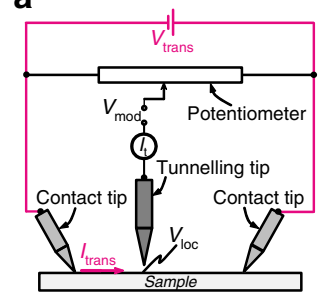

d

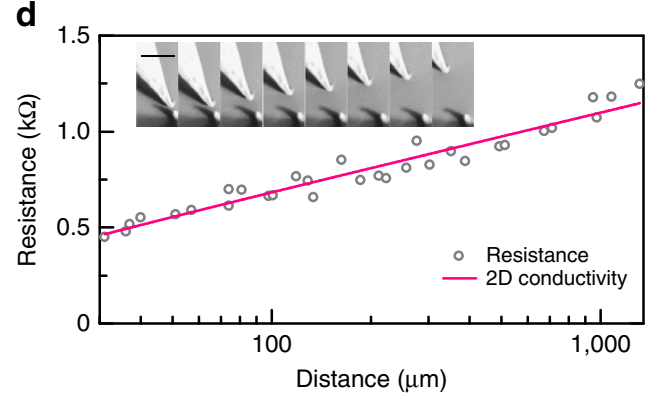

C

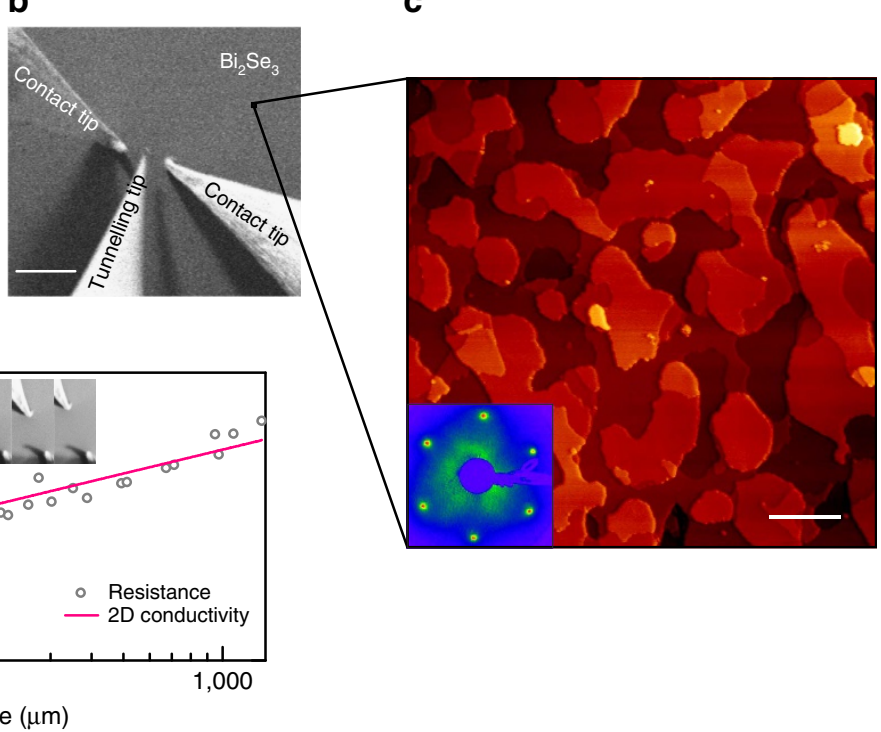

Figure 1 | SEM, STM, LEED and sheet conductance of $\mathbf{B i}_{\mathbf{2}} \mathbf{S e}_{\mathbf{3}}$ on $\mathbf{S i ( 1 1 1 ) . ~ ( a ) ~ S c h e m e ~ o f ~ t h e ~ S T P ~ e x p e r i m e n t : ~ a ~ v o l t a g e ~} V_{\text {trans }}$ leading to a transverse current $I_{\text {trans }}$ is applied to the sample by two tips (red circuit). A potentiometer connects the tunnelling tip to the sample contacts in a Wheatstone bridge circuit. $V_{\text {loc }}$ represents the local potential at the surface under the tunnelling tip. (b) SEM image of the contact geometry. Scale bar, $75 \mu \mathrm{m}$. Two Au tips contact the $\mathrm{Bi}_{2} \mathrm{Se}_{3}$ sample surface. A W tip is operated in tunnelling distance (tunnelling tip) and simultaneously maps the surface structure and the electrochemical potential. (c) STM image of the $14 \mathrm{QL} \mathrm{Bi}_{2} \mathrm{Se}_{3}$ film on $\mathrm{Si}(111)\left(V_{\mathrm{t}}=1 \mathrm{~V}, \mathrm{I}_{\mathrm{t}}=12 \mathrm{pA}, 650 \times 650 \mathrm{~nm}^{2}\right)$. Scale bar, $100 \mathrm{~nm}$. The surface of the Bi $\mathrm{Se}_{3}$ film is crystalline and flat. The inset shows the hexagonal LEED pattern of the $\mathrm{Bi}_{2} \mathrm{Se}_{3}$ film at $35 \mathrm{eV}$ (lattice constant of $0.41 \pm 0.01 \mathrm{~nm}$ ) exhibiting the high crystallinity of the film. (d) Distance-dependent resistance measurement of the $\mathrm{Bi}_{2} \mathrm{Se}_{3}$ film, leading to a sheet conductance of $1.8 \pm 0.1 \mathrm{mS}$. The inset shows SEM image snapshots of the measuring process. Scale bar, $75 \mu \mathrm{m}$.

Excluding tip induced artefacts in the STP measurements. However, a drop in the measured potential may be caused by the geometry of the tip (that is, a double tip as discussed, for example, by ref. 21): In this case, the tunnelling junction is laterally displaced, leading to a voltage drop $\Delta V$ of the potential given by $E \cdot d$ with the electric field $E$ and the distance $d$ for the double tip. For the given values of $E$ and $\Delta V$, the distance would be $\sim 7 \mathrm{~nm}$. Hence, the deviation of the constant gradient of the potential should be limited to a range of $\sim 7 \mathrm{~nm}$. However, we find that the change of the potential at a step edge remains for distances in the order of $100 \mathrm{~nm}$ or more. This has been further corroborated by a numerical simulation taking care of different tip geometries and the measured topography. Here, it is worth noting, that the measured STP signals at the $\mathrm{Bi}_{2} \mathrm{Se}_{3}$ step edges are very small, reaching the limits of the STP measurement. Similar signals observed for the graphene surface (see ref. 28 for details) and the $\operatorname{Si}(111)-(\sqrt{3} \times \sqrt{3}) \mathrm{R}^{\circ} 0^{\circ}-\mathrm{Ag}$ surface $^{29}$ are a magnitude higher $(\mathrm{mV}$ scale) than in our case ( $\mu \mathrm{V}$ scale). Therefore, great care was taken to exclude artefacts. For a detailed discussion, see the Supplementary Note 3 inclusive Supplementary Figs 3 and 4.

Such a sharp voltage drop at a surface step edge is only observed in STP, if the underlying scattering event is located extremely close to the surface (M. Wenderoth and R. Möller, personal communication). Thus, the observed small voltage drops at surface step edges of $\mathrm{Bi}_{2} \mathrm{Se}_{3}$ clearly reveal that significant surface contributions are measured by our STP experiment and we conclude that a significant charge transport is mediated through the TI surface at room temperature.

Detailed STP analysis at a 1 QL step. To analyse the voltage drop at a 1 QL step in more detail, Fig. 3a shows averaged line profiles of the potential across a $1 \mathrm{QL} \mathrm{Bi}_{2} \mathrm{Se}_{3}$ step for different surface current densities. The values of the observed voltage drops at the step edge are stated at each line profile. To improve the signal to noise ratio, we average the data for several scan lines (see also the Supplementary Note 4 and Supplementary Figs 5 and 6). By evaluating the voltage drop at the single QL step edge as a function of the surface current density $\left(j_{\mathrm{s}}=-70 \mathrm{~mA} \mathrm{~cm}^{-1}\right.$ $\ldots+70 \mathrm{~mA} \mathrm{~cm}^{-1}$, Fig. 3b) we can deduce the conductivity at a QL step edge. As expected, for the low surface-current densities, the amplitude of the voltage drop scales linearly as a function of the surface current density. Now we compare an average local voltage drop of $30 \mu \mathrm{V}$ with the observed gradient for a length of $100 \mathrm{~nm}$, which is equivalent to a voltage difference of $720 \mu \mathrm{V}$. Using the fact that $30 \%$ of the current density flows through the surface state $e^{9,10,12}$, we estimate a voltage difference for a nominal length of $100 \mathrm{~nm}$ caused by phonon scattering to $210 \mu \mathrm{V}$ for a pure surface conduction. Thus, the voltage drop at the step edge accounts to $15 \%$ for a length of $100 \mathrm{~nm}$, which is a significant contribution to the surface state conductivity and will be even higher for an increased step edge density. Similar to the evaluation of the step conductivity induced by a substrate step of graphene on $\mathrm{SiC}$ in ref. 28, we determine the step conductivity of a $1 \mathrm{QL} \mathrm{Bi} \mathrm{Se}_{3}$ step from the slope of the fit to a numerical value of $\sigma_{\text {step }}=j_{\mathrm{s}} / \Delta V=1,100 \pm 700 \mathrm{~S} \mathrm{~cm}^{-1}$ (Fig. 3b). The main factors for the uncertainties for the step conductivity are the measured distances between the two contact tips (25\%) and the estimation of the portion of transvers current, which flows through the surface state $(33 \%)$. Other error sources like the uncertainties in the measurement of the transverse current and in the measurements of the voltage drops are less important (some \%). This leads to a total relative error of the step conductivity of $\sim 60 \%$. From other STP data on $\mathrm{Bi}_{2} \mathrm{Se}_{3}$ (see Supplementary Note 5 and Supplementary Fig. 7), we can also make a rough estimation of the step conductivity of a 3 QL step to a numerical value of about $400 \mathrm{~S} \mathrm{~cm}^{-1}$. The impact of surface step edges to the lateral film conductance was also verified by macroscopic 4 point probe measurements. Here, we used a $\mathrm{Bi}_{2} \mathrm{Se}_{3}$ film with an anisotropic step distribution and find that the conductance of the film is also anisotropic. The resulting evaluation of the step edge 
conductivity from those macroscopic measurements yields a value of $1,000 \mathrm{~S} \mathrm{~cm}^{-1}$ for the step conductivity of the $\mathrm{Bi}_{2} \mathrm{~S}_{3}$ steps in great agreement with the STP measurements. For more details, see the Supplementary Note 6 and Supplementary Figs 8-10. a

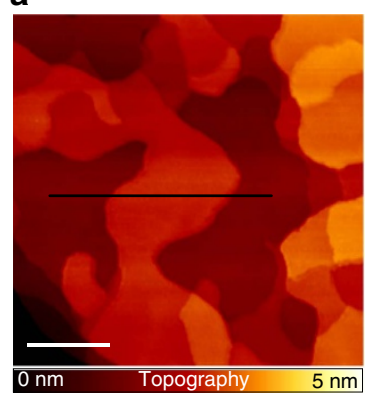

c

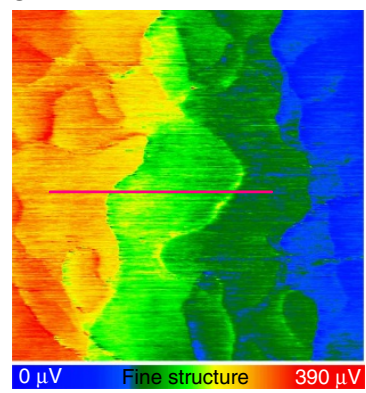

b

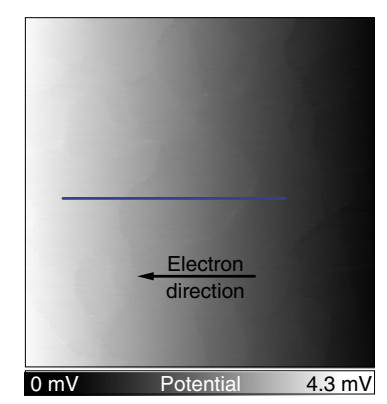

d

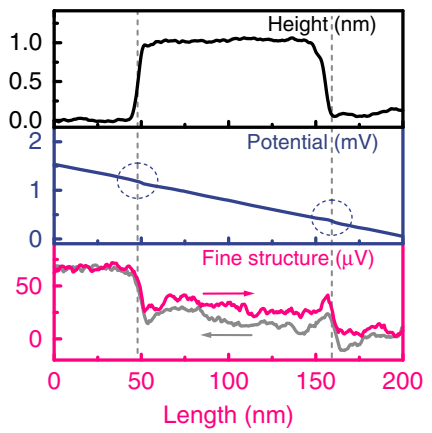

Figure 2 | Results of the STP measurement. $\left(V_{\bmod }=20 \mathrm{mVpp}\right.$ at $2.1 \mathrm{kHz}$, $I_{\mathrm{t}}=12 \mathrm{pA}, V_{\text {trans }}=-1.3 \mathrm{~V}, I_{\text {trans }}=-1.9 \mathrm{~mA}, 315 \times 315 \mathrm{~nm}^{2}$ ). White scale bar, $75 \mathrm{~nm}$. (a) Topography of the $\mathrm{Bi}_{2} \mathrm{Se}_{3}$ film. (b) The image of the potential for the corresponding surface area as in a mainly exhibits a constant gradient corresponding to the electric field induced by the contacting tips. (c) Corresponding 'fine structure' of the potential (gradient subtracted). The colour coding helps to identify rather sharp drops of the potential at surface step edges. (d) Averaged line profiles (about 7 scan lines) across a $\mathrm{Bi}_{2} \mathrm{Se}_{3}$ island for the same position as marked in $\mathbf{a}, \mathbf{b}$ and $\mathbf{c}$. The plot of the fine structure includes the profile for the backward scan direction (light grey). The scan direction is indicated by arrows.

\section{Discussion}

The observed voltage drops demonstrate that the step edges are nano-resistances, which scatter the conduction electrons locally. This scattering process can be described as a reflection and transmission of incoming electron waves at the step edge, which does not contradict to the properties of a TI. Very recent ab-initio calculations by Narayan et al. ${ }^{19}$ show that $\mathrm{Bi}_{2} \mathrm{Se}_{3}$ step edges are barriers for the electron transport, which is consistent with our STP results. The direct backscattering $\left(180^{\circ}\right)$ is still forbidden, but other scattering channels (angles) are possible as discussed by Biswas and Balatzky ${ }^{15}$. This was already observed indirectly by $\mathrm{d} I / \mathrm{d} V$ imaging, for example, on $\mathrm{Bi}_{1-x} \mathrm{Sb}_{x}$ by Roushan et al. ${ }^{4}$, on the $\mathrm{Bi}_{2} \mathrm{Te}_{3}$ surface by Alpichshev et al. ${ }^{5}$ and on the $\mathrm{Bi}_{2} \mathrm{Se}_{3}$ surface by Wang et al. ${ }^{6}$ Depending on the electron energy, the phase and the spin texture, the scattered electron waves interfere at the step edge with the incoming or transmitted electron waves resulting in oscillations of the LDOS near the step edge ${ }^{14,16}$, which diminish faster (power of $-3 / 2$ ) than for trivial two dimensional electron gas systems (power of -1/2) (ref. 6). Although direct backscattering for the surface state is not allowed, other non$180^{\circ}$ scattering channels are available at the step edge, which limit the resulting conductivity of the $\mathrm{Bi}_{2} \mathrm{Se}_{3}$ surface. Here, it is worth noting that we cannot clearly distinguish if the scattering happens exclusively within the TI's surface state or if bulk states ${ }^{30}$ also participate in the scattering process. Epitaxial $\mathrm{Bi}_{2} \mathrm{Se}_{3}$ films are known to exhibit a n-doping because of Se vacancies, leading to a shift of the Fermi energy towards the valence band. At a high

\section{Table 1 | Step conductivity $\sigma_{\text {step }}$ of different 2D systems measured by STP*.}

\begin{tabular}{|c|c|}
\hline System & $\sigma_{\text {step }}\left(\mathrm{S} \mathrm{cm}^{-1}\right)$ \\
\hline Si(111)- $(\sqrt{3} \times \sqrt{3})$ R30-Ag (Si step $)^{29}$ & $32 \pm 5$ \\
\hline $\mathrm{Si}(111)-(\sqrt{3} \times \sqrt{3}) \mathrm{R} 30^{\circ}-\mathrm{Ag}$ (Si multiple step) $)^{29}$ & $7 \pm 3$ \\
\hline Graphene on SiC (monolayer on a SiC step) ${ }^{28}$ & $1,400 \pm 500$ \\
\hline Graphene on $\mathrm{SiC}$ (monolayer on a SiC double step) ${ }^{28}$ & $700 \pm 200$ \\
\hline Graphene on $\mathrm{SiC}$ (monolayer on a SiC triple step) ${ }^{28}$ & $400 \pm 100$ \\
\hline $\mathrm{Bi}_{2} \mathrm{Se}_{3}$ (1 QL step) & $1,100 \pm 700$ \\
\hline $\mathrm{Bi}_{2} \mathrm{Se}_{3}$ (3 QL step) & ca 400 \\
\hline
\end{tabular}
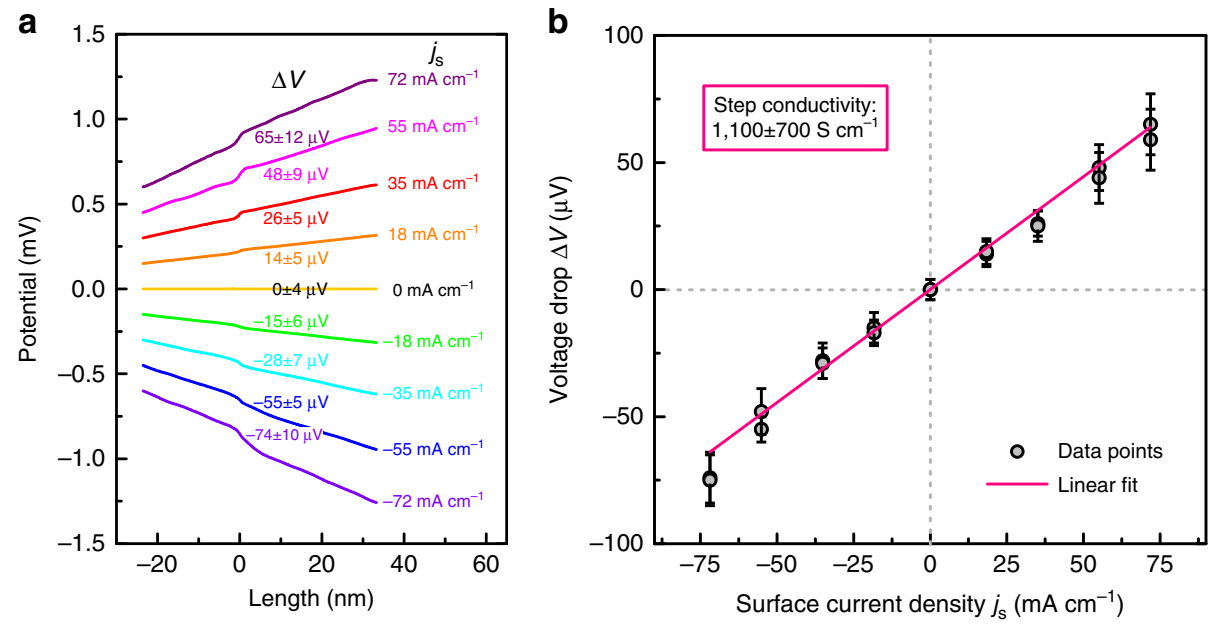

Figure 3 | Step conductivity of a 1 QL step. (a) Averaged line profile of the potential in the vicinity of a 1-QL step of $\mathrm{Bi}_{2} \mathrm{Se}_{3}$ for different surface current densities $j_{\mathrm{s}}$ from $-72 \mathrm{~mA} \mathrm{~cm}^{-1}$ to $72 \mathrm{~mA} \mathrm{~cm}^{-1}$. For clarity, the line profiles are vertically offset. The value of the voltage drop $\Delta V$ at the step edge is indicated. (b) Voltage drop at a 1-QL step as a function of surface current density. The linear fit yields the conductivity of a 1-QL step to 1,100 $\pm 700 \mathrm{~S} \mathrm{~cm}{ }^{-1}$. The individual error bars of the voltage drops represent the maximal error of each measured voltage drop as estimated from the noise level. 
doping level, this would lead to an increased involvement of the bulk states in the scattering process. In realistic thin-film devices, such effects are also of technological relevance. Overall, our experimental results reveal that electron scattering also affects the local potential near a surface step edge of the $3 \mathrm{D}$ TI $\mathrm{Bi}_{2} \mathrm{Se}_{3}$ if a current is flowing through the surface. This is the first direct evidence for the relation between LDOS oscillations and local voltage drops at defect sites on TI surfaces.

Table 1 gives an overview on the step conductivities including other low-dimensional surface systems. The step conductivity of a $1-\mathrm{QL} \mathrm{Bi}_{2} \mathrm{Se}_{3}$ step (height of ca $1 \mathrm{~nm}$ ) is similar to a $\mathrm{SiC}$ substrate step for a graphene layer ${ }^{28}$ (height of $0.5 \mathrm{~nm}$ ). If we compare steps of the same step height (graphene on a $\mathrm{SiC}$ double step versus 1 $\mathrm{QL} \mathrm{Bi}_{2} \mathrm{Se}_{3}$ step), the step conductivity of $\mathrm{Bi}_{2} \mathrm{Se}_{3}$ appears enhanced. This means that the impact of the step edge scattering in the case of $\mathrm{Bi}_{2} \mathrm{Se}_{3}$ thin films is reduced as compared with a substratesupported graphene sheet. In comparison to, for example, a single step of the $\mathrm{Si}(111)-(\sqrt{ } 3 \times \sqrt{3}) \mathrm{R} 30^{\circ}-\mathrm{Ag}$ (ref. 29) surface (height of $0.3 \mathrm{~nm}$ ), the step conductivity of a $1-\mathrm{QL} \mathrm{Bi}_{2} \mathrm{Se}_{3}$ step appears 35 times higher. This implies that the overlap of the surface states on the upper and the lower terrace at the $\mathrm{Bi}_{2} \mathrm{Se}_{3}$ step edge is quite high. This is reasonable as the penetration depth of the surface state extents about 3 QL (ref. 23).

In conclusion, voltage drops at step edges of the $\mathrm{Bi}_{2} \mathrm{Se}_{3}$ surface reveal elementary contribution to the resistivity of TI surfaces. This proves that despite the topological protection the surface morphology plays a critical role for the electron transport, for example, in prospective thin TI film-based devices. For an ideal TI without any bulk contributions to the conductivity, the sum of the nano-resistances at step edges will limit the macroscopic surface conductivity. Our approach can also be applied to analyse scattering of conduction electrons at other defects structures such as domain boundaries, non-magnetic and magnetic adsorbates. With this knowledge, it should be possible to tune the electron transport of a TI surface on a local scale for designing smaller and more complex nanoscale device structures.

\section{Methods}

Sample preparation of $\mathbf{B i}_{2} \mathbf{S e}_{3}$ thin films. For the preparation of $\mathrm{Bi}_{2} \mathrm{Se}_{3}$ films, we follow the recipe of Zhang et al. ${ }^{24}$ and Vyshnepolsky et al. ${ }^{31}$ :

Bi (purity of $99.997 \%$ by Mateck) and Se (purity of $99.999 \%$ by Mateck) are coevaporated with a ration Bi:Se of 1:2.25 (excess of Se) onto a $\mathrm{Si}(111)-(\sqrt{3} \times \sqrt{3})$-Bi substrate at room temperature ${ }^{24}$. The amount of deposited material is monitored by a quartz micro balance. The geometrical structure for the different steps of preparation is checked by LEED. A Si wafer with a low n-doping (phosphorus, conductivity of $7.7 \mathrm{mS} \mathrm{cm}^{-1}$ ) and a miscut of $0.5^{\circ}$ (ca $30 \mathrm{Si}$-steps $\mu \mathrm{m}^{-1}$ ) was used. Before the deposition of $\mathrm{Bi}_{2} \mathrm{Se}_{3}$, the wafer was flashed to $1,500 \mathrm{~K}$ and slowly cooled to induce the $7 \times 7$ reconstruction. The equivalent of $10 \mathrm{ML}$ of bismuth was deposited at a sample temperature of $300 \mathrm{~K}$ followed by heating to $720 \mathrm{~K}$ to prepare the $\mathrm{Bi}-(\sqrt{3} \times \sqrt{3})$-reconstruction. We grew a $\mathrm{Bi}_{2} \mathrm{Se}_{3}$ film with a nominal film thickness of ca $14 \mathrm{~nm}$ (about $14 \mathrm{QL}$ ), which is known to exhibit the topological phase $^{23}$. Finally, the sample is annealed at $530 \mathrm{~K}$ for $2 \mathrm{~h}$ to ensure a flat and smooth film morphology 31 .

Scanning tunnelling potentiometry. We use STP (Fig. 1a), which was first introduced by Muralt and Pohl ${ }^{21}$ in 1986. This is a STM-related technique, which allows us to measure the local electrochemical potential $\mu_{\mathrm{ec}}$ and the local topography of the sample with atomic resolution simultaneously. In brief, two tips contact the sample and apply a voltage $V_{\text {trans }}$ leading to a transverse current $I_{\text {trans }}$ through the surface. A third tip is brought into tunnelling distance in the area between both contact tips. A potentiometer connects the contact tips and the tunnelling tip in a Wheatstone bridge circuit. If the bridge is balanced, the average DC tunnelling current $I_{\mathrm{t}}$ vanishes $\left(\left\langle I_{\mathrm{t}}\right\rangle=0\right)$ and the voltage at the tip matches the local potential $V_{\text {loc }}$ at the position of the tunnelling tip. During scanning, the bridge is automatically readjusted for each position of the tunnelling tip and the voltage at the tip is recorded (see Bannani et al. ${ }^{22}$ for details). In addition, a small alternating bias $V_{\text {mod }}$ is applied to the tunnelling junction resulting in an AC tunnelling current, which is used to control the distance between tip and sample. Thus, topographic STM imaging and potential mapping are simultaneously provided by STP. The geometry of the three tips and the sample surface monitored by SEM is shown in Fig. 1b.

\section{References}

1. Hasan, M. Z. \& Kane, C. L. Topological insulators. Rev. Mod. Phys. 82, 3045-3067 (2010).

2. Zhang, H., Liu, C.-X., Dai, X., Fang, Z. \& Zhang, S.-C. Topological insulators in $\mathrm{Bi}_{2} \mathrm{Se}_{3}, \mathrm{Bi}_{2} \mathrm{Te}_{3}$ and $\mathrm{Sb}_{2} \mathrm{Te}_{3}$ with a single Dirac cone on the surface. Nature Phys. 5, 438-442 (2009).

3. Mann, C. et al. Mapping the $3 \mathrm{D}$ surface potential in $\mathrm{Bi}_{2} \mathrm{Se}_{3}$. Nat. Commun 4, 2277 (2013).

4. Roushan, P. et al. Topological surface states protected from backscattering by chiral spin texture. Nature 460, 1106-1109 (2009).

5. Alpichshev, Z. et al. STM imaging of electronic waves on the surface of $\mathrm{Bi}_{2} \mathrm{Te}_{3}$ : topologically protected surface states and hexagonal warping effects. Phys. Rev. Lett. 104, 016401 (2010).

6. Wang, J. et al. Power-law decay of standing waves on the surface of topological insulators. Phys. Rev. B 84, 235447 (2011).

7. Lang, M. et al. Revelation of topological surface states in $\mathrm{Bi}_{2} \mathrm{Se}_{3}$ thin films by in situ Al passivation. ACS Nano 6, 295-302 (2012).

8. Hor, Y. S. et al. p-type $\mathrm{Bi}_{2} \mathrm{Se}_{3}$ for topological insulator and low-temperature thermoelectric applications. Phys. Rev. B 79, 195208 (2009).

9. He, L. et al. Epitaxial growth of $\mathrm{Bi}_{2} \mathrm{Se}_{3}$ topological insulator thin films on $\mathrm{Si}$ (111). J. Appl. Phys. 109, 103702 (2011).

10. He, L. et al. Surface-dominated conduction in a $6 \mathrm{~nm}$ thick $\mathrm{Bi}_{2} \mathrm{Se}_{3}$ thin film. Nano Lett. 12, 1486-1490 (2012).

11. Kim, D. et al. Surface conduction of topological Dirac electrons in bulk insulating $\mathrm{Bi}_{2} \mathrm{Se}_{3}$. Nat. Phys. 8, 459-463 (2012).

12. Steinberg, H., Gardner, D. R., Lee, Y. S. \& Jarillo-Herrero, P. Surface state transport and ambipolar electric field effect in $\mathrm{Bi}_{2} \mathrm{Se}_{3}$ nanodevices. Nano Lett. 10, 5032-5036 (2010).

13. Teague, M. L. et al. Observation of Fermi-energy dependent unitary impurity resonances in a strong topological insulator $\mathrm{Bi}_{2} \mathrm{Se}_{3}$ with scanning tunneling spectroscopy. Solid State Commun. 152, 747-751 (2012).

14. Cottin, M. C. et al. Interplay between forward and backward scattering of spin-orbit split surface states of $\mathrm{Bi}(111)$. Nano Lett. 13, 2717-2722 (2013).

15. Biswas, R. R. \& Balatzky, A. V. Scattering from surface step edges in strong topological insulators. Phys. Rev. B 83, 075439 (2011).

16. Jing, W. \& Bang-Fen, Z. Elastic scattering of surface states on threedimensional topological insulators. Chin. Phys. B 22, 067301 (2013).

17. Zhang, T., Levy, N., Ha, J., Kuk, Y. \& Stroscio, J. A. Scanning tunneling microscopy of gate tunable topological insulator $\mathrm{Bi}_{2} \mathrm{Se}_{3}$ thin films. Phys. Rev. B 87, 115410 (2013)

18. Alos-Palop, M., Tiwari, R. P. \& Blaaboer, M. Suppression of conductance in a topological insulator nanostep junction. Phys. Rev. B 87, 035432 (2013).

19. Narayan, A., Rungger, I., Droghetti, A. \& Sanvito, S. Ab-initio transport across Bismuth Selenide surface barriers. Phys. Rev. B 90, 205431 (2014).

20. Datta, S. in Electronic Transport in Mesoscopic Systems 72 (Cambridge Univ., 1997).

21. Muralt, P. \& Pohl, D. W. Scanning tunneling potentiometry. Appl. Phys. Lett. 48, 514-516 (1986).

22. Bannani, A., Bobisch, C. A. \& Möller, R. Local potentiometry using a multiprobe scanning tunneling microscope. Rev. Sci. Instrum 79, 083704 (2008).

23. $\mathrm{Wu}, \mathrm{L}$. et al. A sudden collapse in the transport lifetime across the topological phase transition in $\left(\mathrm{Bi}_{1-x} \operatorname{In}_{x}\right)_{2} \mathrm{Se}_{3}$. Nat. Phys 9, 410-414 (2013).

24. Zhang, G. et al. Quintuple-layer epitaxy of thin films of topological insulator $\mathrm{Bi}_{2} \mathrm{Se}_{3}$. Appl. Phys. Lett. 95, 053114 (2009).

25. Taskin, A. A., Sasaki, S., Segawa, K. \& Ando, Y. Manifestation of topological protection in transport properties of epitaxial $\mathrm{Bi}_{2} \mathrm{Se}_{3}$ thin films. Phys. Rev. Lett. 109, 066803 (2012)

26. Butch, N. P. et al. Strong surface scattering in ultrahigh-mobility $\mathrm{Bi}_{2} \mathrm{Se}_{3}$ topological insulator crystals. J. Phys. Rev. B 81, 241301 (2010).

27. Ghaemi, P., Mong, R. S. K. \& Moore, J. E. In-plane transport and enhanced thermoelectric performance in thin films of the topological insulators $\mathrm{Bi}_{2} \mathrm{Te}_{3}$ and $\mathrm{Bi}_{2} \mathrm{Se}_{3}$. Phys. Rev. Lett. 105, 166603 (2010).

28. Ji, S. H. et al. Atomic-scale transport in epitaxial graphene. Nat. Mater 11, 114 (2011).

29. Homoth, J. et al. Electronic transport on the nanoscale: ballistic transmission and Ohm's Law. Nano Lett. 9, 1588 (2009).

30. Kim, S. et al. Surface scattering via bulk continuum states in the $3 \mathrm{D}$ topological insulator $\mathrm{Bi}_{2} \mathrm{Se}_{3}$. Phys. Rev. Lett. 107, 056803 (2013).

31. Vyshnepolsky, M., Klein, C., Klasing, F., Hanisch-Blicharski, A. \& Horn-von Hoegen, M. Epitaxial growth of the topological insulator $\mathrm{Bi}_{2} \mathrm{Se}_{3}$ on $\mathrm{Si}(111)$ : growth mode, lattice parameter, and strain state. Appl. Phys. Lett. 103, 111909 (2013).

\section{Acknowledgements}

We gratefully acknowledge Rolf Möller for fruitful discussions and also for the possibility to perform the experiments with the four probe STM. Especially the STP simulation as provided in the supporting information bases on his contribution. Special thanks go also to Michael Horn-von Hoegen, M. Wenderoth and Nicolás Lorente for stimulating and 
supporting discussions. We also thank the German Research Council (DFG) for funding through project BO 3427/2-1. D. Utzat is gratefully acknowledged for designing and constructing the STM and STP electronics.

\section{Author contributions}

The manuscript was written by contributions from both the authors. S.B. and C.A.B. planned the experiments and discussed on the analysis of the data. S.B. performed the measurements and C.A.B. conceived the experiment and supervised the work. All authors have given approval to the final version of the manuscript.

\section{Additional information}

Supplementary Information accompanies this paper at http://www.nature.com/ naturecommunications
Competing financial interests: The authors declare no competing financial interest.

Reprints and permission information is available online at http://npg.nature.com/ reprintsandpermissions/

How to cite this article: Bauer, S. and Bobisch, C. A. Nanoscale electron transport at the surface of a topological insulator. Nat. Commun. 7:11381 doi: 10.1038/ncomms11381 (2016).

\section{(c) (1)}

This work is licensed under a Creative Commons Attribution 4.0 International License. The images or other third party material in this article are included in the article's Creative Commons license, unless indicated otherwise in the credit line; if the material is not included under the Creative Commons license, users will need to obtain permission from the license holder to reproduce the material. To view a copy of this license, visit http://creativecommons.org/licenses/by/4.0/ 